\title{
PREDICTIVE ROLE OF CDX2, MUC2 AND MUC6 IN PROGRESSION OF CHRONIC GASTRITIS TO CARCINOMA
}

\author{
Assaf M.I, El Gohary Y. M, Zaher T. I and Atwa H.A. \\ Department of Pathology, Faculty of Medicine, Zagazig University.
}

\begin{abstract}
Background and aim: The material of this study comprises 57 gastric biopsies including paraffin blocks selected from the archives of Pathology Department, Faculty of medicine, Zagazig University in addition to 10 freshly randomly selected endoscopic biopsies were obtained from the Endoscopy Unit of Tropical Medicine department, Faculty of Medicine, Zagazig University hospital in the period from 2011-2013 to examine the expression of CDX2,MUC2 and MUC6 in cases of chronic gastritis, gastric metaplasia, gastric dysplasia and cancer and study the role of these markers as predictors of progression of chronic gastritis to carcinoma.

Methods: Immunohistochemical analysis of CDX2, MUC2 and MUC6 was performed using 50 cases for chronic superficial gastritis, chronic atrophic gastritis, chronic atrophic gastritis with intestinal metaplasia, gastric dysplasia and gastric cancer.

Results: CDX2 and MUC2 were negative in 11/11 cases of chronic superficial and chronic atrophic gastritis on the other hand, CDX2 was positive in $3 / 3$ cases of complete intestinal metaplasia while CDX2 was positive in 10/12 cases of incomplete intestinal metaplasia. CDX2 was positive in 6/8 cases of low grade dysplasia and 2/4 cases of high grade dysplasia. CDX2 was positive in $62.5 \%$ of cases of gastric adenocarcinoma and was positive in $2 / 2$ cases of mucinous carcinoma. $2 / 2$ of signet ring carcinomas showed negative CDX2 expression. MUC2 was positive in all cases of complete and incomplete IM (3/3 and 12/12 respectively). MUC2 was positive in 7/8 cases of low grade dysplasia and 2/4 cases of high grade dysplasia. MUC2 was positive in $50 \%$ of cases of gastric adenocarcinoma. MUC2 was positive in all studied cases of mucinous carcinoma. 2 /2 of signet ring carcinomas showed negative MUC2 expression. MUC6 was positive in 11/11 cases of chronic superficial and chronic atrophic gastritis. It was positive in 11 cases of incomplete intestinal metaplasia. MUC6 was positive in 1 case of low grade dysplasia and 1case of high grade dysplasia and 5 cases of gastric cancer but all 3 cases of complete intestinal metaplasia showed negative MUC6 expression. MUC6 was positive in $37.5 \%$ of cases of gastric adenocarcinoma and in 2/2 cases of signet ring carcinomas. MUC6 expression was negative in studied 2 cases of mucinous carcinoma.

Conclusion: Gastric carcinomas are classified into gastric and intestinal phenotype on the basis of mucin expression.CDX2 transcriptional factor has a role in the formation and progression of intestinal metaplasia (IM) among the diverse gastric disorders such as gastritis, dysplasia and Gastric cancer(GC). CDX2 may be closely related to IM and The intestinal-type GC. Further loss of CDX2 in incomplete intestinal metaplasia (IM) could be a key step that may precedes the morphological change to dysplasia and may help to predict the risk of gastric cancer.CDX2 and MUC2 can be considered as a marker of intestinal metaplasia. MUC6 can serve as a marker for pyloric metaplasia
\end{abstract}

\section{INTRODUCTION}

G astric cancer is the fourth most common cancer and the second leading cause of cancer-related death worldwide. Gastric cancer is known to increase with age with the peak incidence occurring at 60-80 years. [1]. In Egypt, gastric cancer is in the eleventh rank constituting $2.1 \%$ of all cancers. At National Cancer institute (NCI), CairoUniversity, gastric cancer constituted $1.8 \%$ of all cancers and $10.3 \%$ of gastrointestinal cancers with a median age 53 [2]. Gastric adenocarcinoma, is either intestinal or diffuse. Intestinal-type adenocarcinoma, may be proceeded by precancerous lesions starts by chronic gastritis which progresses to mucosal atrophy (atrophic gastritis) followed by intestinal metaplasia, dysplasia and carcinoma. No preceding steps have been identified in the pathogenesis of diffuse tumor, other than the chronic gastritis associated with Helicobacter pylori infection [3]. The chronic inflammation of the stomach is usually caused by $\mathrm{H}$. pylori infection. The cytokines released during bacterial infection play a vital role in triggering cellular changes that contribute to gastric mucosal damage and gastric neoplastic transformation [4].

Intestinal metaplasia (IM) can be classified into different subtypes by several classification systems. The most widely accepted one is to classify IM into complete type and incomplete type, with the latter carrying a higher risk of gastric cancer especially Type III incomplete IM [5].

Gastric dysplasia is classified as low- or high-grade usually depends on the degree of nuclear atypia and architectural distortion [6].

Mucins are high molecular weight extracellular glycoproteins that maintain epithelial integrity and lubricate and protect epithelial surfaces. Normal gastric mucosa expresses MUC1, MUC5AC and MUC6. MUC2 is generally absent in the normal gastric mucosa [7]

De novo expression of MUC2 appears in areas of intestinal metaplasia and in malignancy [8].

Caudal-related homeobox transcription factor (CDX2), is a member of the caudal-related homeobox gene family, plays an important role in 
mammalian early intestinal development and the maintenance of intestinal epithelia. It is not expressed in normal stomach [9]

Several studies showed CDX2 expression in intestinal metaplasia and many gastric cancer, suggesting that ectopic CDX2 expression may be involved in gastric carcinogenesis [10], [11] and[12].

\section{MATERIAL AND METHODS}

A total of fifty seven cases of gastric lesions and include 24 chronic gastritis (active gastritis, chronic atrophic gastritis), 13 gastric dysplasia (8 low grade dysplasia and 4 high grade dysplasia, 16 chronic gastritis with intestinal metaplasia, 14 gastric carcinoma (GC) classified into ( 2 well differentiated (gradeI) adenocarcinoma, 3 invasive chronic superficial gastritis, chronic moderately differentiated adenocarcinoma (grade II), 2 poorly differentiated adenocarcinoma (grade III), 2 mucinous adenocarcinoma and 2 signet ring carcinoma).These specimens were received by pathology department, faculty of medicine, Zagazig university in the period from September 2011 to September 2013 and from National Cancer Institute, Cairo University, in the period from 2011 to 2013. In addition ten fresh randomly selected endoscopic biopsies were obtained from the Endoscopy Unit of Tropical Medicine department, Faculty of Medicine, Zagazig University hospital in the period from 2011-2013. These fresh specimens were fixed in formalin, dehydrated in ascending grades of alcohol, cleared in xylene and embedded in paraffin blocks. All tissue samples were formalin-fixed and paraffinembedded. Hematoxylin and eosin (H\&E) stained slides and pathologic reports were reviewed to confirm the diagnosis as well as to diagnose 10 fresh specimens.

\section{Immunohistochemical staining:}

Among all 67 cases of specimen examined, only 50 representative cases for chronic superficial gastritis, chronic atrophic gastritis, chronic atrophic gastritis with intestinal metaplasia, gastric dysplasia and gastric cancer were submitted for Immunohistochemical evaluation owing to insufficient specimens in the remaining (17 cases). Paraffin sections 3-5 um were deparaffinized in the oven at $56{ }^{\circ} \mathrm{C}$ for 30 minutes, and inserted in xylene for 30 minutes. Tissues were rehydrated in descending grades of alcohol $95 \%, 85 \%$ and then $75 \%$ for 5 minutes each. Slides were rinsed with distilled water for 5 minutes. Antigen retrieval was performed by boiling in sodium citrate buffer $(0.001 \mathrm{M}, \mathrm{pH} 6)$ for 15 minutes in microwave. Endogenous peroxidase activity was blocked by incubation with hydrogen peroxide for 10 minutes. Then rinse with distilled water.

Apply primary monoclonal rabbit anti-cdx2 antibody (Cat. from Thermo Scientific/Lab Vision Corporation, Fermont, USA, and clone: EPR2764. $0.09 \%$ sodium azide. Dilution 1:100), mouse monoclonal antibody anti muc2 antibody (mouse monoclonal antibody Cat. from Thermo Scientific/Lab Vision Corporation, Fermont, USA and clone: M53. 0.09\% sodium azide. Dilution 1:50) and rabbit polyclonal muc6 (Cat. from Thermo Scientific/Lab Vision Corporation, Fermont, USA, $0.09 \%$ sodium azide.Dilution 1:100) overnight at $4^{\circ} \mathrm{C}$. After 3 wash with PBS and sections were incubated with biotinylated secondary antibodies at for $30 \mathrm{~min}$. This is followed by incubation with streptavidin-biotinperoxidase complex. After 3 rinses with PBS, The slides were incubated with diaminobenzidinefor 15 min. The slides were rinsed with $\mathrm{H} 2 \mathrm{O}$ and counerstained with hematoxylin for 3 minutes. This was followed by washing in cold running water, then wash in distilled water. Sections were dehydrated in ascending grades of alcohol and cleared with xylene, then coverslipped and examined.

Positive control for CDX2 was cancer colon. The negative control was obtained by omission of the primary antibody.

Positive controls for MUC2 were taken from colon or stomach. The negative control was obtained by omission of the primary antibody.

Positive controls for MUC6 were taken from stomach. The negative control was obtained by omission of the primary antibody.

Scoring Criteria: Both qualitative and semiquantitative approaches were used in scoring the staining of MUC2, MUC6 and CDX2. Samples were classified as positive if $>5 \%$ cells stained positive and otherwise as negative.

Semi-quantitative scores were given as the score of the percentage of positive cells plus the score of the staining intensity. The scoring criteria of the percentage of positive cells score 0: 0\%-5\% positive cells; score 1: $6 \%-25 \%$ positive cells; score 2: $26 \%-50 \%$ positive cells; score 3: $51 \%$ $75 \%$ positive cells; and score 4: $76 \%-100 \%$ positive cells. The intensity was scored as follows: score 0 , no staining; score 1 , weak/equivocal staining; score 2, mild staining; score 3, moderate staining; score 4, strong staining [13].

Statistical analysis: Statistical analysis was performed using SPSS software. The degree of correlation between different parameters was evaluated by using the chi- square test. A 
difference of $P<0.05$ between groups was considered significant.

\section{RESULTS}

CDX2 was positive in 3 studied cases of complete intestinal metaplasia, 10 cases of complete intestinal metaplasia, 6 of cases of low grade dysplasia, 2 cases of high grade dysplasia and 7 cases of gastric adenocarcinoma. Negative CDX2 expression in all 11 studied cases of chronic superficial and chronic atrophic gastritis, table (1).Positive CDX2 was highly significant in complete IM, incomplete IM and low grade dysplasia $(\mathrm{p}=0.001)$. CDX2 expression was significantly negative in chronic superficial and chronic atrophic gastritis $(\mathrm{p}=0.001)$.

MUC2 was positive in 3 studied cases of complete intestinal metaplasia, 12 studied cases of incomplete intestinal metaplasia, 7 cases of low grade dysplasia, 2 cases of high grade dysplasia and 6 cases of gastric adenocarcinoma. Negative MUC2 expression in all 11 studied cases of chronic superficial and chronic atrophic gastritis, table (2).

Positive MUC2 was significant in incomplete metaplasia and high dysplasia ( $\mathrm{p}=0.004$ and 0.02 ) respectively. MUC2 expression was significantly negative in chronic superficial and chronic atrophic gastritis $(\mathrm{p}=0.001)$.

MUC6 was positive in 11 studied cases of chronic superficial and chronic atrophic gastritis, 11 cases of incomplete intestinal metaplasia.
MUC6 was positive in 1 case of low grade dysplasia and 1case of high grade dysplasia and 5 cases of gastric cancer. Negative MUC6 expression in all 3 studied cases of complete intestinal metaplasia, table (3)

Positive MUC6 was highly significant in chronic superficial gastritis, chronic atrophic gastritis and incomplete metaplasia p $(=0.001)$. Positive MUC6 was significant in low grade dysplasia $(\mathrm{p}=0.015)$.

No significant relationship was found between adenocarcinoma, signet and mucinous carcinoma as regarding their IHC reactivity to CDX2, MUC2 and MUC6 ( $\mathrm{p}=0.11,0.13$ and 0.11) respectively

No significant difference was found between well, moderately and poorly differentiated gastric adenocarcinoma as regarding their IHC reactivity to CDX2, MUC2 and MUC6 ( $\mathrm{p}=0.19,0.71$ and $0.19)$ respectively.

\section{CDX2 and MUC2 Immunoprofile of gastric lesion}

$(\mathrm{CDX} 2+/ \mathrm{muc} 2+)$ immunoprofile was positive in cases of 3 studied cases of complete intestinal metaplasia, 10 cases of incomplete intestinal metaplasia, 8 cases of gastric dysplasia and 4 cases of gastric cancer.

(CDX2-/muc2-) immunoprofile was observed in 11 studied cases of chronic superficial and atrophic gastritis. It was statistically significant, table (4)

Table (1): IHC expression pattern of CDX2 in gastric lesions

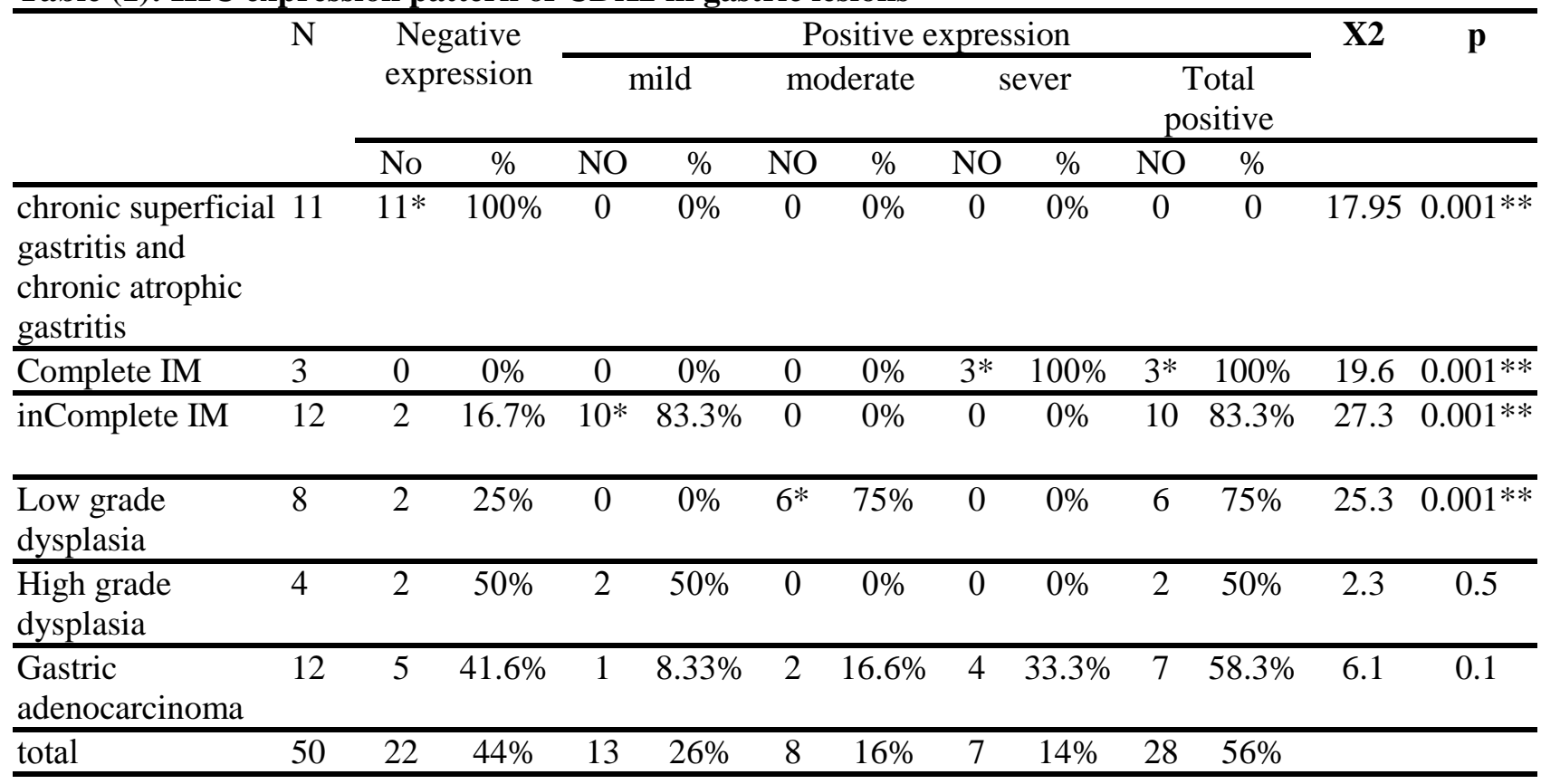


Table (2): IHC expression pattern of MUC2 in gastric lesions

\begin{tabular}{|c|c|c|c|c|c|c|c|c|c|c|c|c|c|}
\hline & \multirow[t]{3}{*}{$\mathrm{N}$} & \multirow{2}{*}{\multicolumn{2}{|c|}{$\begin{array}{c}\text { Negative } \\
\text { expression }\end{array}$}} & \multicolumn{6}{|c|}{ Positive expression } & \multirow{2}{*}{\multicolumn{2}{|c|}{$\begin{array}{c}\text { Total } \\
\text { positive }\end{array}$}} & \multirow[t]{3}{*}{$\overline{X 2}$} & \multirow[t]{3}{*}{$\mathrm{p}$} \\
\hline & & & & \multicolumn{2}{|c|}{ mild } & \multicolumn{2}{|c|}{ moderate } & \multicolumn{2}{|c|}{ sever } & & & & \\
\hline & & $\mathrm{No}$ & $\%$ & $\mathrm{No}$ & $\%$ & $\mathrm{No}$ & $\%$ & $\mathrm{No}$ & $\%$ & $\mathrm{NO}$ & $\%$ & & \\
\hline $\begin{array}{l}\text { Chronic } \\
\text { superficial } \\
\text { gastritis and } \\
\text { chronic atrophic } \\
\text { gastritis }\end{array}$ & 11 & 11 & 100 & 0 & 0 & $\overline{0}$ & 0 & $\overline{0}$ & $\overline{0}$ & 0 & & 21.15 & $0.001 * *$ \\
\hline Complete IM & 3 & 0 & $0 \%$ & 0 & $0 \%$ & 0 & $0 \%$ & 3 & $100 \%$ & 3 & $100 \%$ & 3.46 & 0.17 \\
\hline inComplete IM & 12 & 0 & $0 \%$ & 0 & $0 \%$ & 2 & $16.7 \%$ & 10 & $83.3 \%$ & 12 & $100 \%$ & 10.7 & $0.004^{*}$ \\
\hline $\begin{array}{l}\text { Low grade } \\
\text { dysplasia }\end{array}$ & 8 & 1 & $12.5 \%$ & $\overline{0}$ & $0 \%$ & 1 & $12.5 \%$ & 6 & $75 \%$ & $\overline{7}$ & $87.5 \%$ & 3.25 & 0.19 \\
\hline $\begin{array}{l}\text { High grade } \\
\text { dysplasia }\end{array}$ & 4 & 2 & $50 \%$ & $\overline{0}$ & $0 \%$ & 2 & $50 \%$ & 0 & $0 \%$ & 2 & $50 \%$ & 7.4 & $0.02 *$ \\
\hline $\begin{array}{l}\text { Gastric } \\
\text { adenocarcinoma }\end{array}$ & 12 & 6 & $50 \%$ & $\overline{0}$ & $0 \%$ & 1 & $8.33 \%$ & $\overline{5}$ & $41.6 \%$ & 6 & $50 \%$ & 0.7 & 0.7 \\
\hline total & 50 & 20 & $40 \%$ & 0 & $0 \%$ & 6 & $12 \%$ & 24 & $48 \%$ & 30 & $60 \%$ & & \\
\hline
\end{tabular}

Table (3): IHC expression pattern of MUC6 in gastric lesions

\begin{tabular}{|c|c|c|c|c|c|c|c|c|c|c|c|c|c|}
\hline & \multirow[t]{3}{*}{$\mathrm{n}$} & \multirow{2}{*}{\multicolumn{2}{|c|}{$\begin{array}{c}\text { Negative } \\
\text { expression }\end{array}$}} & \multicolumn{6}{|c|}{ Positive expression } & \multirow{2}{*}{\multicolumn{2}{|c|}{ Total positive }} & \multirow[t]{3}{*}{$\mathbf{X 2}$} & \multirow[t]{3}{*}{$\mathrm{p}$} \\
\hline & & & & \multicolumn{2}{|c|}{ Mild } & \multicolumn{2}{|c|}{ moderate } & \multicolumn{2}{|c|}{ sever } & & & & \\
\hline & & $\mathrm{n}$ & $\%$ & $\mathrm{n}$ & $\%$ & $\mathrm{n}$ & $\%$ & $\mathrm{n}$ & $\%$ & $\mathrm{NO}$ & $\%$ & & \\
\hline $\begin{array}{l}\text { chronic } \\
\text { superficial } \\
\text { gastritis and } \\
\text { atrophic gastritis }\end{array}$ & 11 & 0 & 0 & 0 & 0 & 0 & $0 \%$ & $11 *$ & 100 & 11 & $100 \%$ & 29.9 & $0.001 * *$ \\
\hline Complete IM & 3 & 3 & $100 \%$ & 0 & $0 \%$ & 0 & $0 \%$ & 0 & $0 \%$ & 0 & $0 \%$ & 4.4 & 0.11 \\
\hline Incomplete IM & 12 & 1 & $8.33 \%$ & 0 & $0 \%$ & $9 *$ & $75 \%$ & 2 & $16.6 \%$ & 11 & $91.7 \%$ & 20.0 & $0.001 * *$ \\
\hline $\begin{array}{l}\text { Low grade } \\
\text { dysplasia }\end{array}$ & 8 & 7 & $87.5 \% *$ & 0 & $0 \%$ & 0 & $0 \%$ & 1 & $12.5 \%$ & 1 & $12.5 \%$ & 8.3 & $0.015^{*}$ \\
\hline $\begin{array}{l}\text { High grade } \\
\text { dysplasia }\end{array}$ & 4 & 3 & $75 \%$ & 0 & $0 \%$ & 1 & $25 \%$ & 0 & $0 \%$ & 1 & $25 \%$ & 2.52 & 0.28 \\
\hline $\begin{array}{l}\text { Gastric } \\
\text { adenocarcinoma }\end{array}$ & 12 & 7 & $58.3 \%$ & 0 & $0 \%$ & 3 & $25 \%$ & 2 & $16.5 \%$ & 5 & $41.6 \%$ & 2.17 & 0.33 \\
\hline$\overline{\text { total }}$ & 50 & 21 & $42 \%$ & 0 & $0 \%$ & 13 & $26 \%$ & 16 & $32 \%$ & 29 & $58 \%$ & & \\
\hline
\end{tabular}

Table (4): CDX2 and MUC2 Immunoprofile of gastric lesion

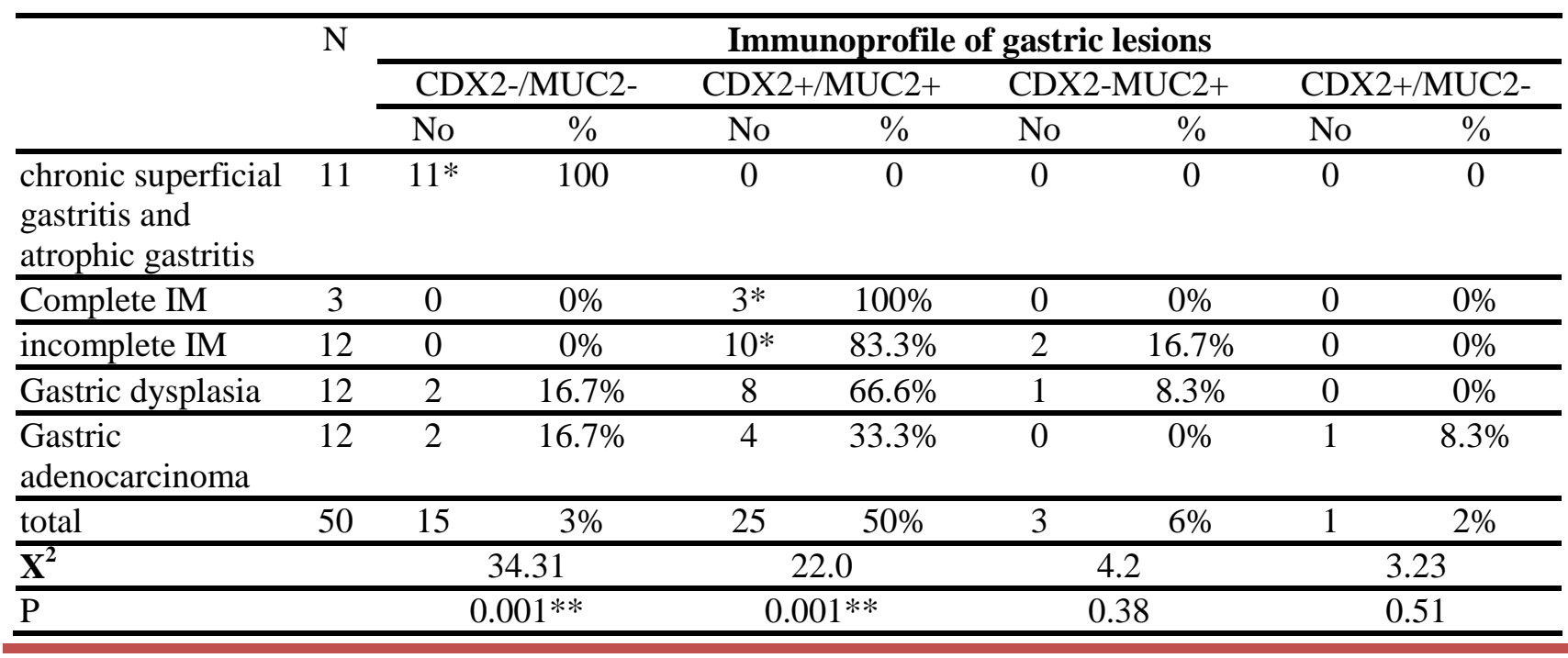




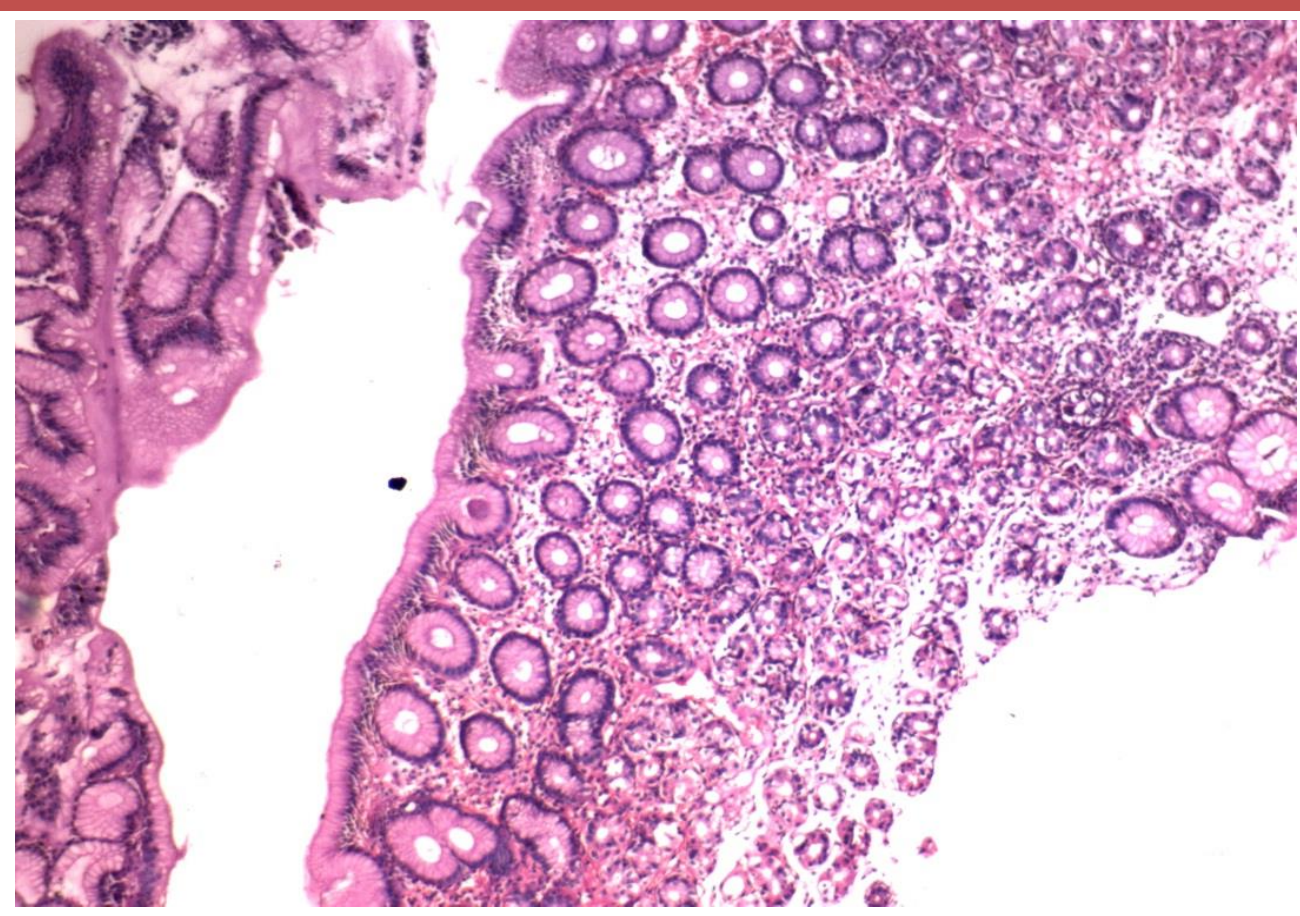

Fig. (1): Chronic superficial gastritis; showing chronic inflammatory cellular infiltrate within the lamina propria.

(Hx\&E X 100).

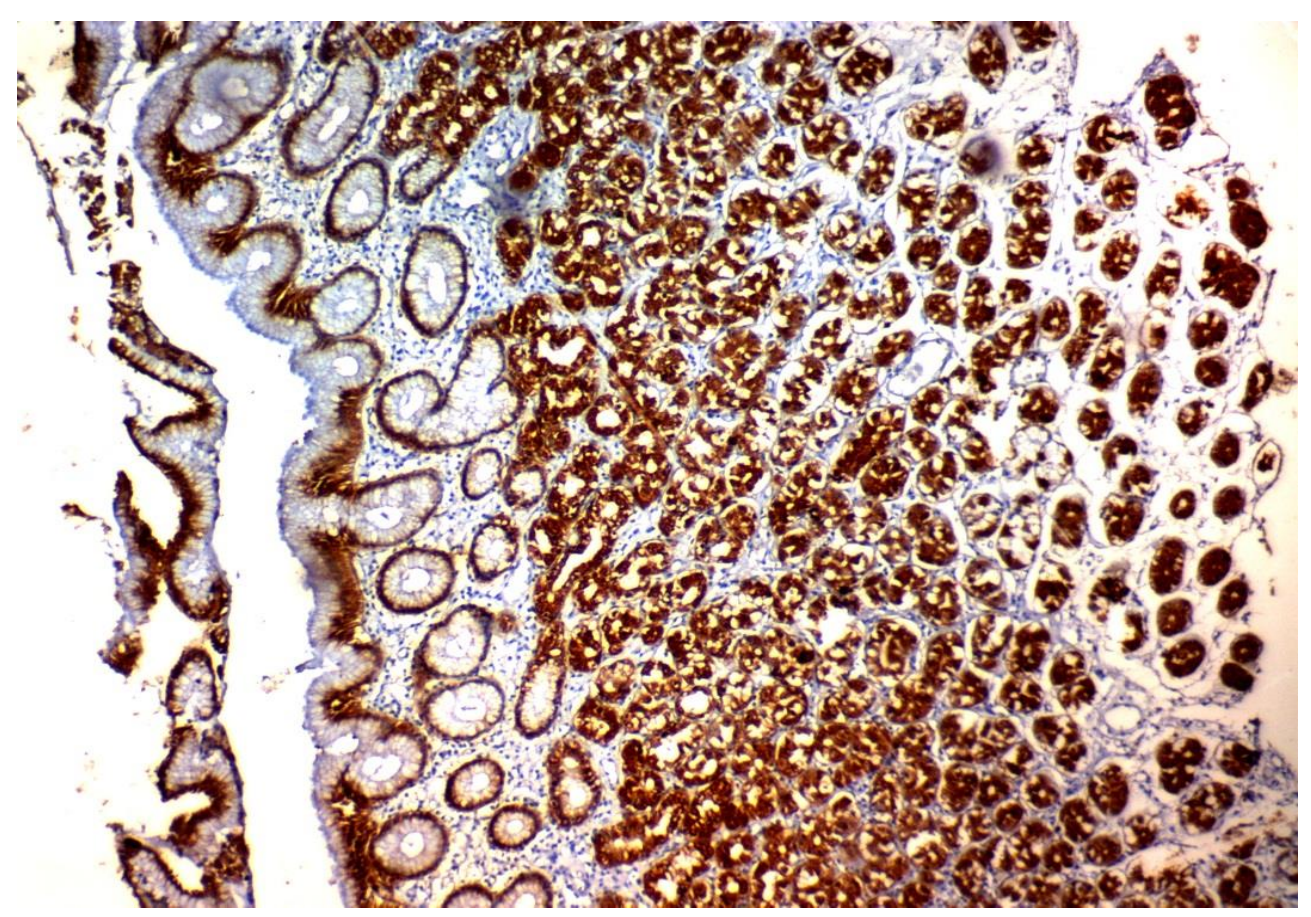

Fig. (2): Chronic superficial gastritis showing strong cytoplasmic immune-reactivity for MUC6. (ABC, DAB x100). 


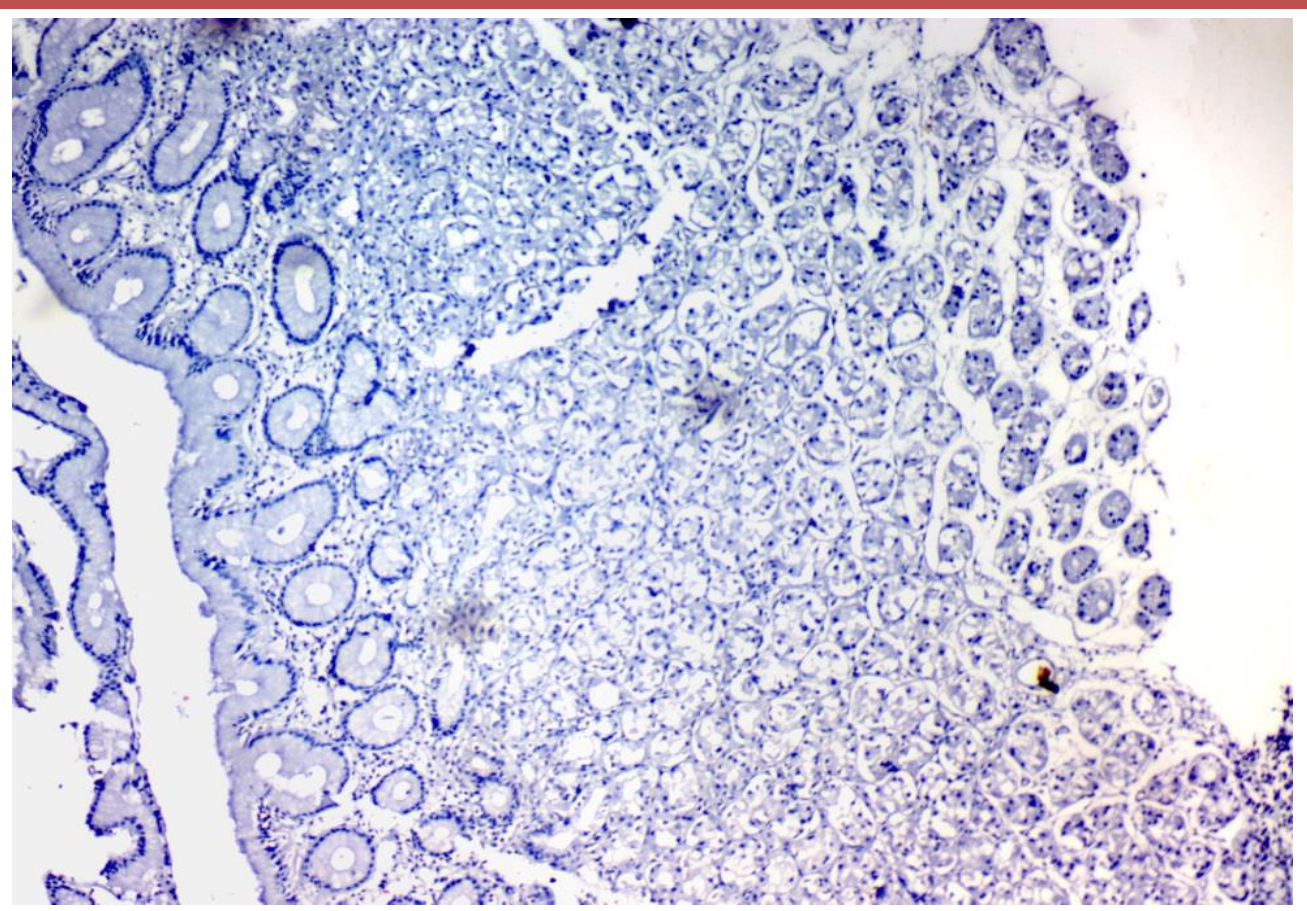

Fig. (3): Chronic superficial gastritis showing negative CDX2 immune-reactivity. (ABC, DAB x100).

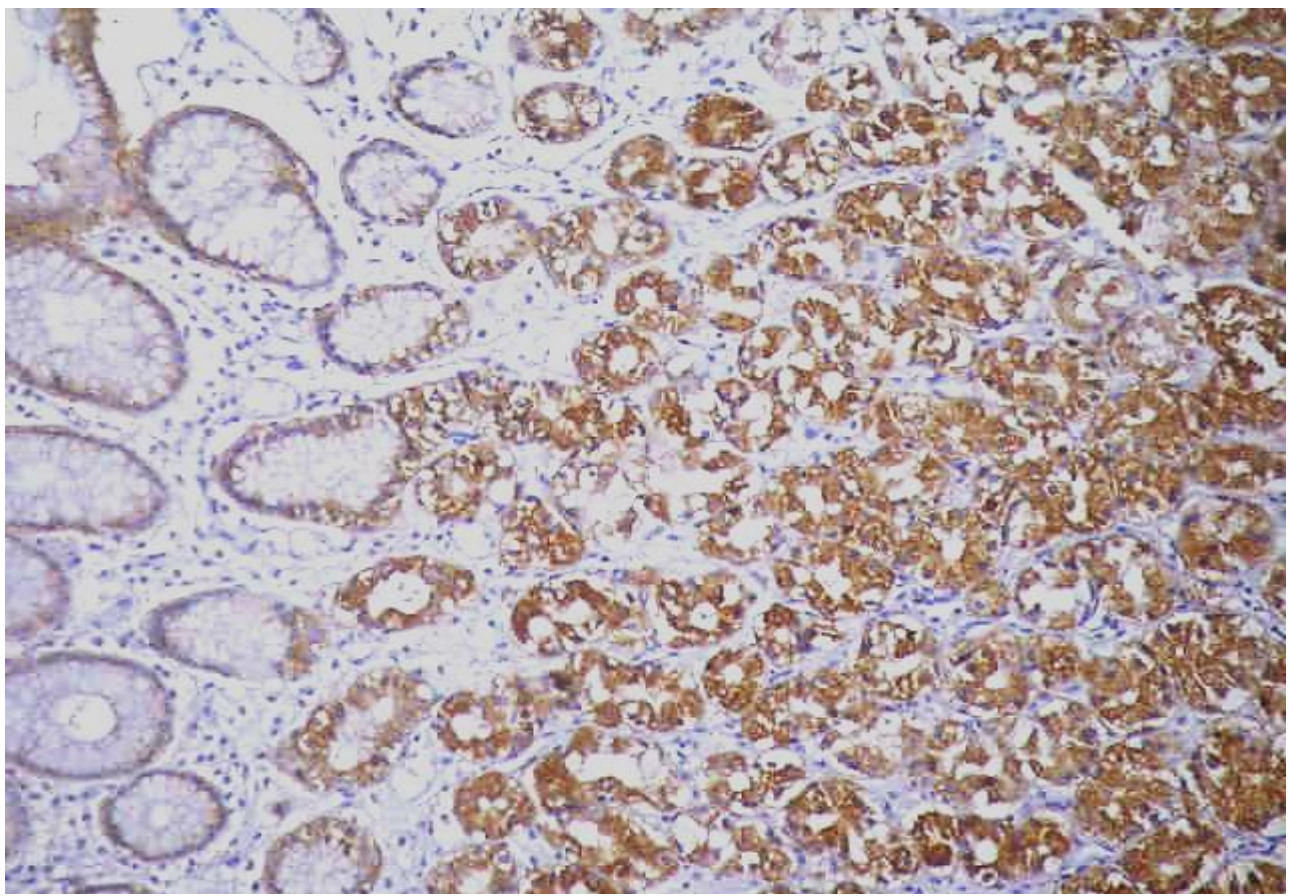

Fig. (5): Chronic atrophic gastritis showing strong cytoplasmic immune-reactivity for MUC6 mainly in pyloric metaplastic glands. (ABC, DAB x100). 


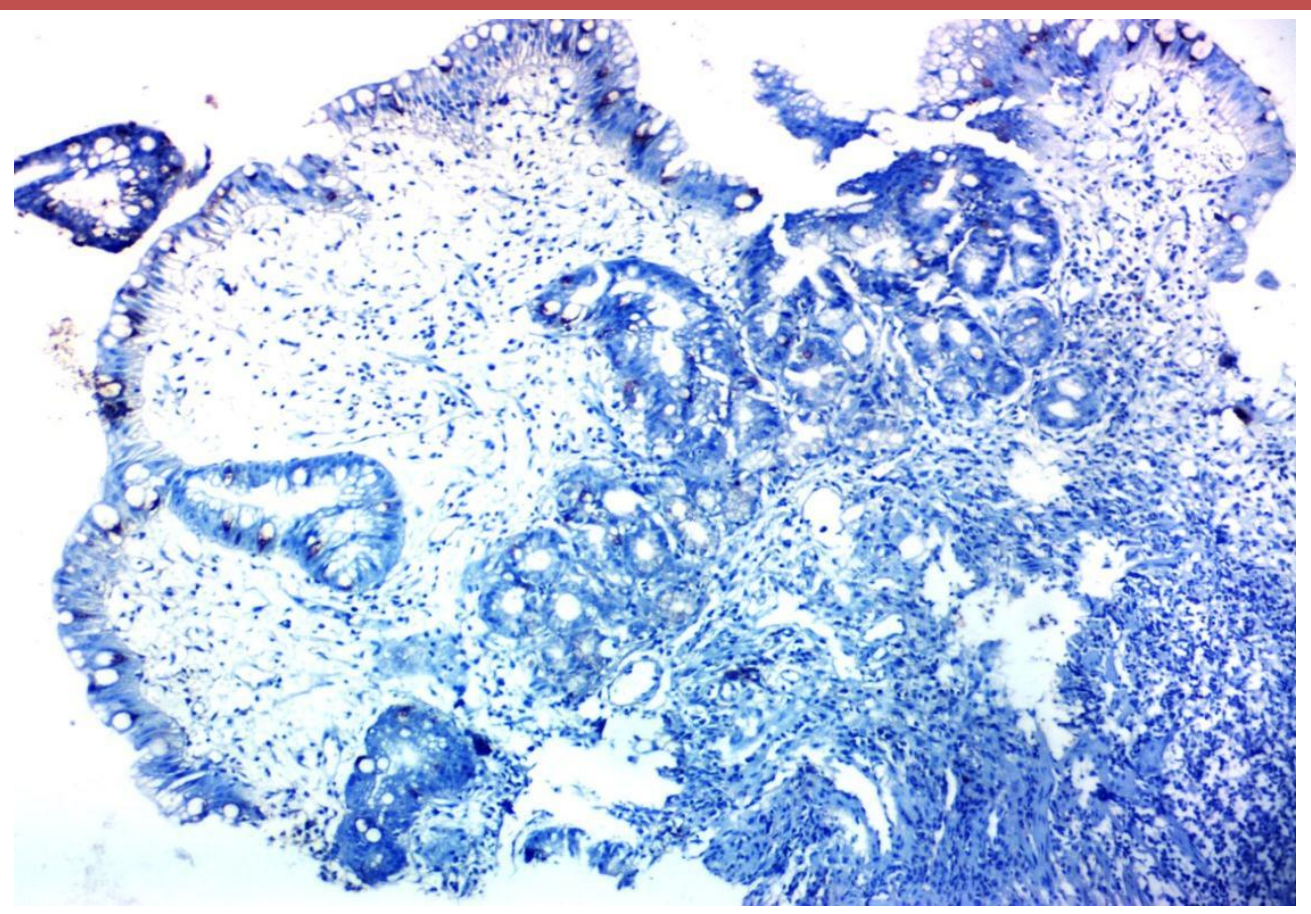

Fig. (6): Chronic atrophic gastritis with incomplete intestinal metaplasia and focal dysplasia showing mild patchy nuclear immune-reactivity for CDX2. (ABC, DAB x100).

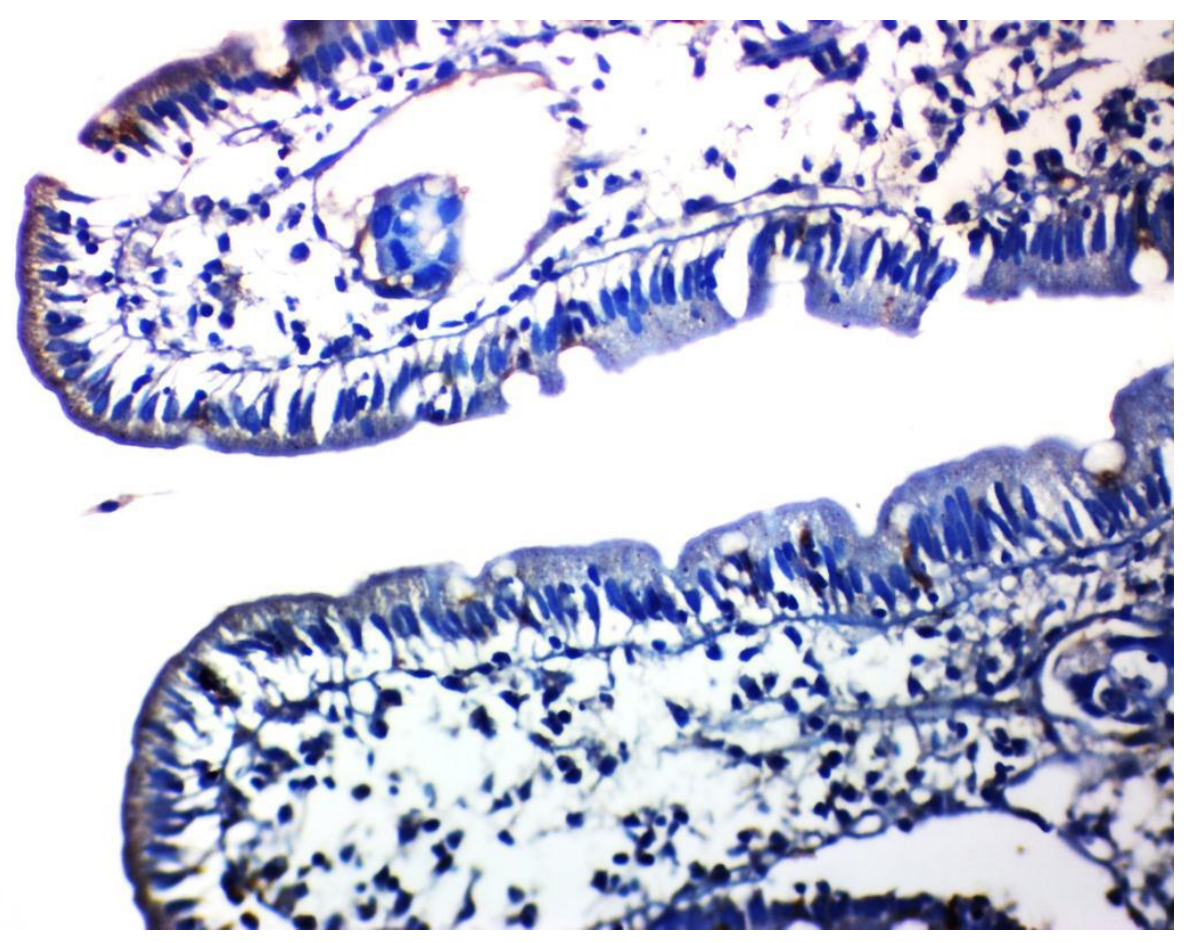

Fig. (7): Chronic atrophic active gastritis with complete intestinal metaplasia showing negative immune-reactivity for MUC6. (ABC, DAB x100). 


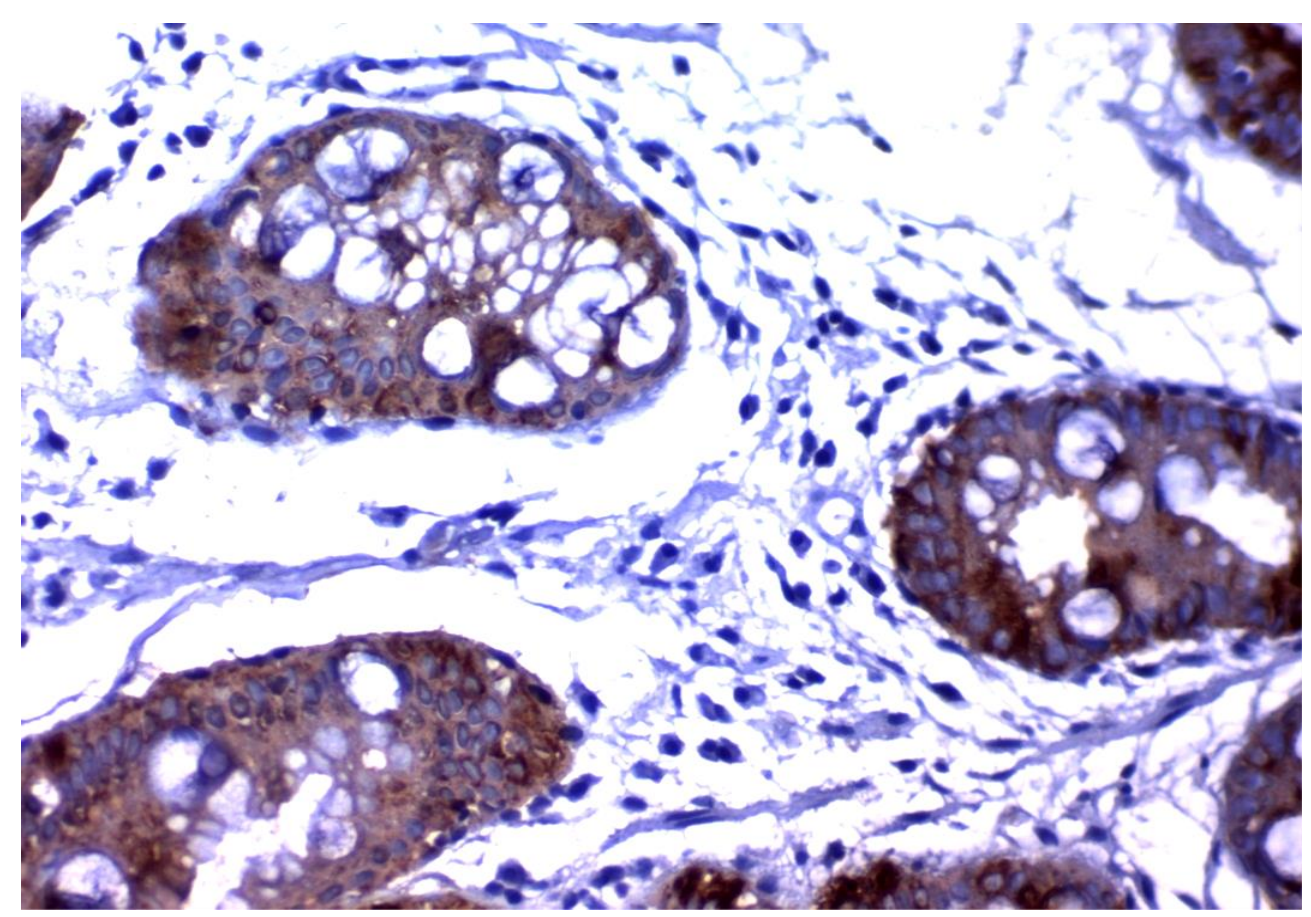

Fig. (8): Chronic atrophic gastritis with incomplete intestinal metaplasia and dysplasia showing strong cytoplasmic immune-reactivity for MUC2. (ABC, DAB x400).

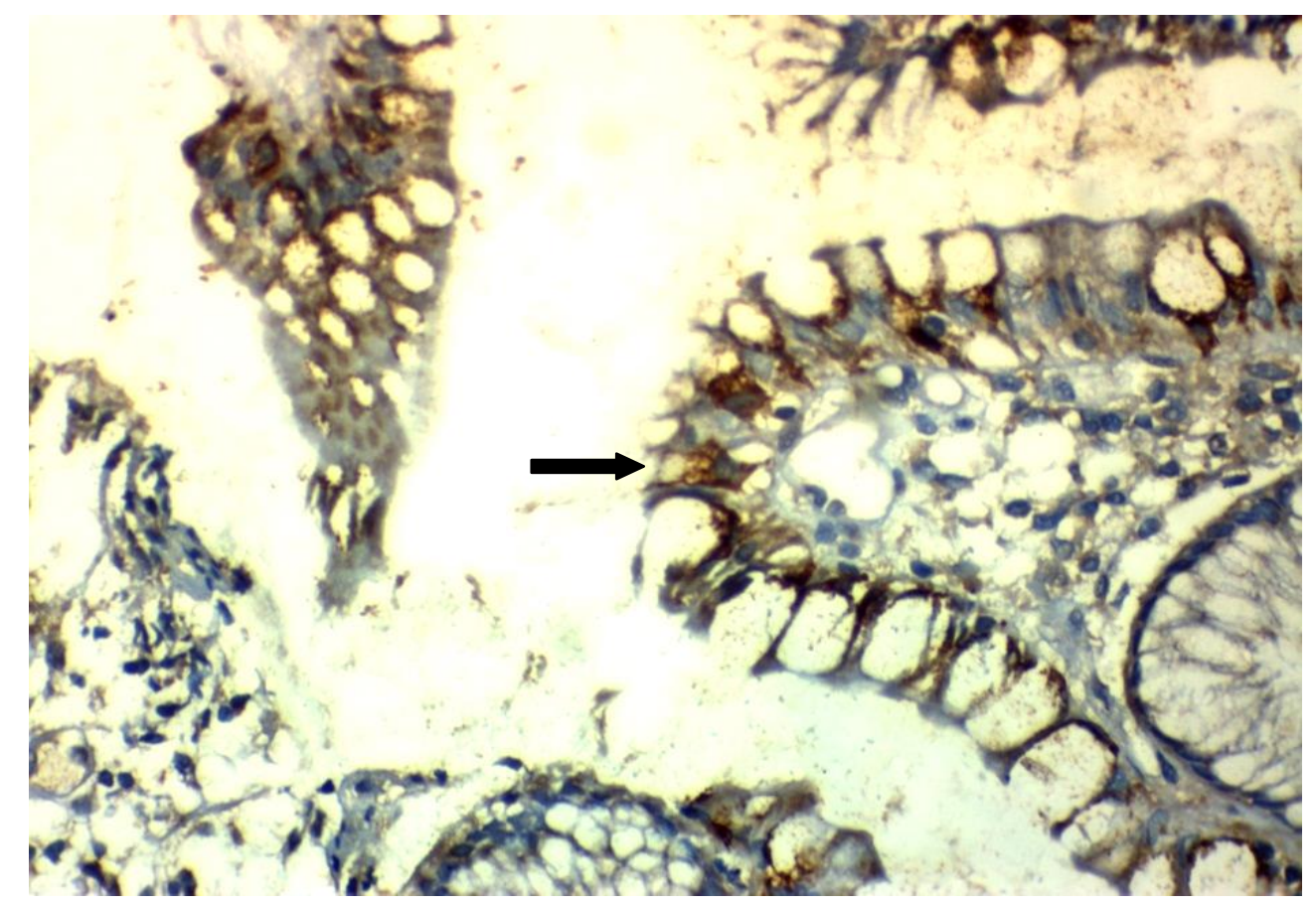

Fig. (10): chronic atrophic active gastritis with complete intestinal metaplasia with intestinal villi showing cytoplasmic and mainly cell membrane immune-reactivity of Goblet cells for MUC2. (Arrow) (ABC, DAB x400). 


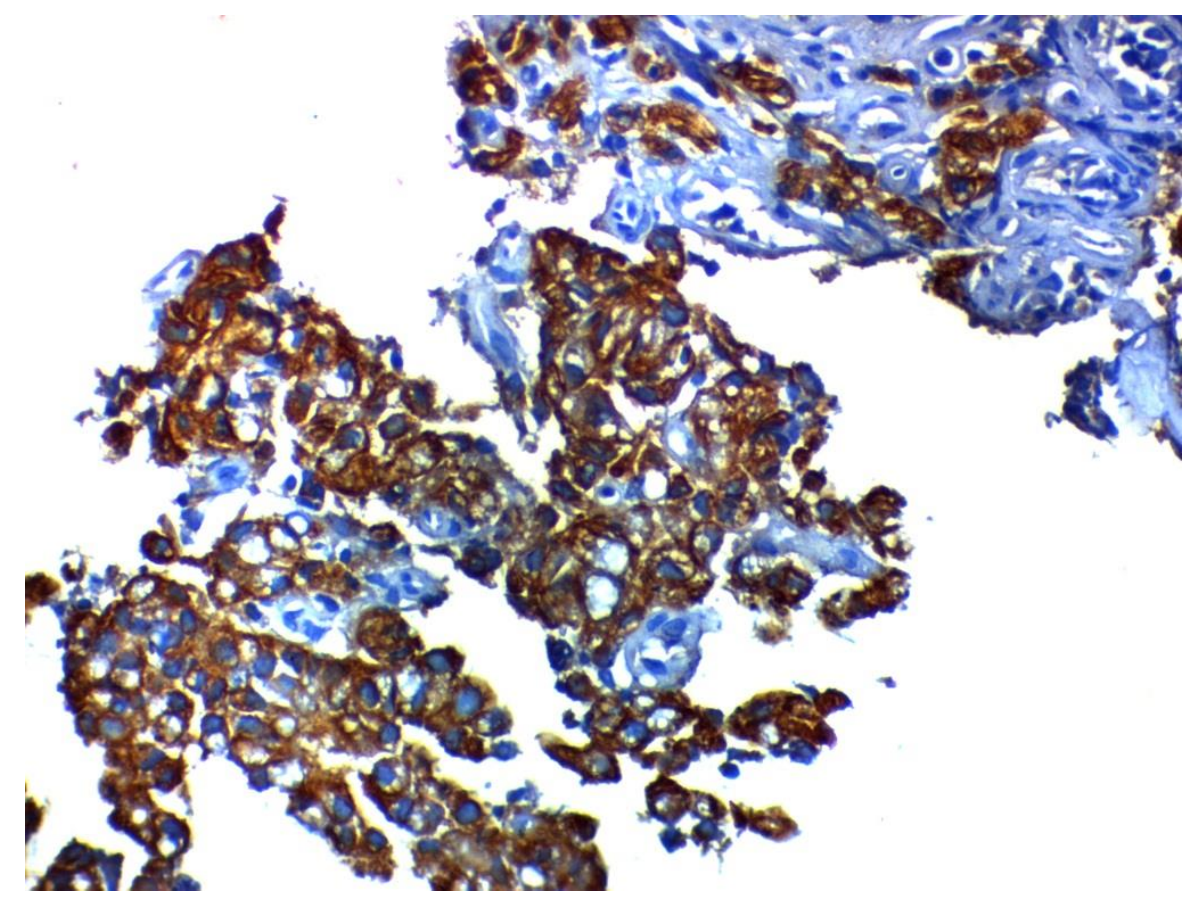

Fig. (9): Signet ring carcinoma showing strong cytoplasmic immune-reactivity for MUC6. (ABC, DAB $\mathrm{x} 400)$.

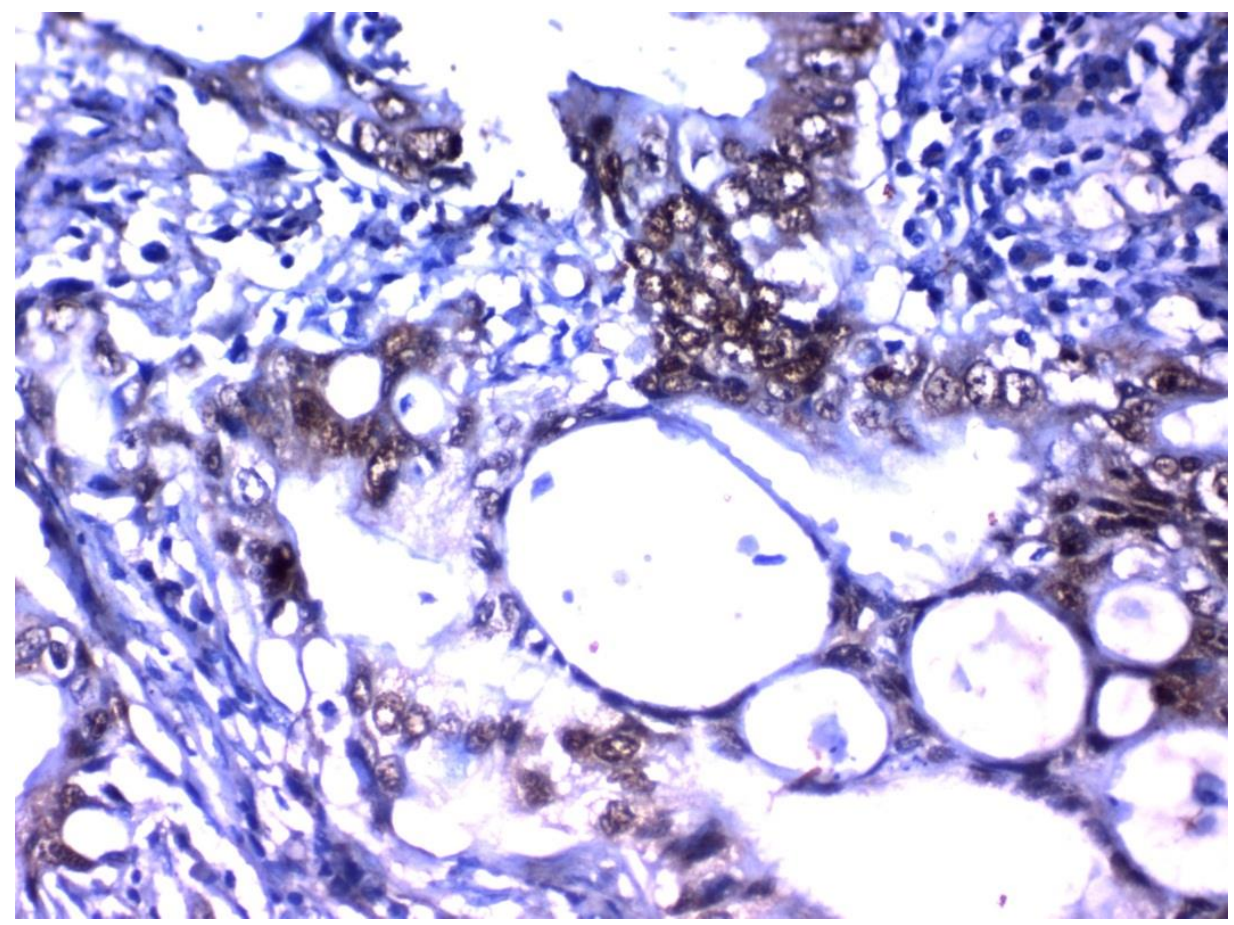

Fig. (10): Well differentiated adenocarcinoma showing strong nuclear immune-reactivity for CDX2. (ABC, DAB $\times 400)$. 


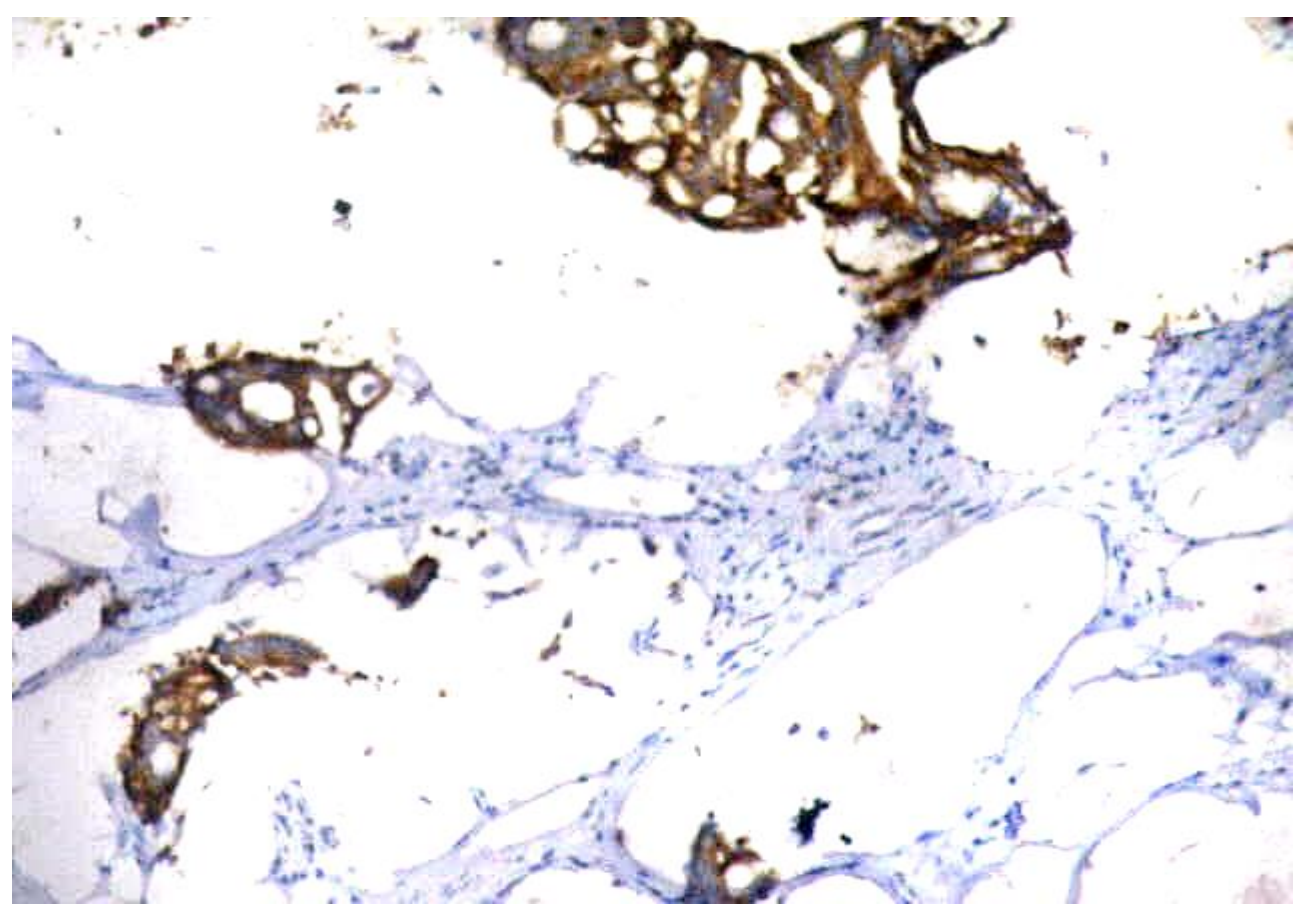

Fig. (11): Mucinous adenocarcinoma showing immune-reactivity for MUC2. (ABC, DAB x100).

\section{DISCUSSION}

In the present study, negative CDX2 expression in all studied 11, this is in agree with RongQin et al. ${ }^{[15]}$ and Liuet al ${ }^{[13]}$.Also in agree with Xin et al. ${ }^{[14]}$ who study the expression of CDX2 and claudin-2 in the multistage tissue of gastric carcinogenesis and found that CDX2positive cases was $0 \%(0 / 108)$ for chronic superficial gastritis, $0 \%(0 / 55)$ for chronic atrophic gastritis.

In the present study, CDX2 was expressed in intestinal metaplasia (IM) in about $86.6 \%(13 / 15)$ and there was immmunohistochemicalexpression difference between CDX2 expressions in complete metaplasia compared to incomplete metaplasia where; the expression in complete IM was $100 \%(3 / 3)$ showing strong nuclear expression and $83.3 \%(10 / 12)$ in incomplete IM showing mild nuclear expression. Positive CDX2 was statistically significant (higher) in complete than incomplete IM, this goes with Liu et al. ${ }^{[13]}$ who demonstrated the expression of CDX2 in all cases of complete IM and this expression was significantly decreased in incomplete IM compared with complete IM. Also in agree with Xin et al. ${ }^{[14]}$ who found CDX2 expression in 90.83\% (99/109) for incomplete-type metaplasia. RongQin et $\mathbf{a l}^{\left[{ }^{[15]}\right.}$ also reported that CDX2 expression in $87.1 \%(101 / 116)$ and found CDX2 expression was $90 \%, 85 \%$ and $75 \%$, respectively in type I, II and type III IM, but the difference was not statistically significant $(\mathrm{P}>0.05)$.

The results are close to seno et al. ${ }^{[12]}$ showing strong nuclear expression and $83.3 \%$ $(10 / 12)$ in incomplete IM showing mild nuclear expression. Positive CDX2 was statistically significant (higher) in complete than incomplete IM, this goes withLiu et al. ${ }^{[13]}$ who demonstrated the expression of CDX2 in all cases of complete IM and this expression was significantly decreased in incomplete IM compared with complete IM. Also in agree with Xin et al. ${ }^{[14]}$ who found CDX2 expression in $90.83 \%$ (99/109) for incomplete-type metaplasia. RongQin et al. ${ }^{[15]}$ also reported that CDX2 expression in $87.1 \%$ (101/116) and found CDX2 expression was 90\%, $85 \%$ and $75 \%$, respectively in type I, II and type III IM, but the difference was not statistically significant $(\mathrm{P}>0.05)$.

The results are close to seno et al. ${ }^{[12]}$ who found CDX2 expression in 89\% (16/18) of intestinal metaplasia. Similar results were reported by Li-Jun Xiao et al. ${ }^{[16]}$ who study CDX2 and villin expression in gastric precancerous lesions and found CDX2 expression in $76.0 \%(19 / 25)$ of IM. 
The results also goes with Almeida et al. ${ }^{[17]}$ who study the putative involvement of two intestine-specific transcription factors, CDX1 and $\mathrm{CDX} 2$, in the pathogenesis of gastric IM and gastric carcinoma and found that $\mathrm{CDX} 2$ was expressed in $94.4 \%$ (17/18) foci of IM.

The results are close to results obtained byLiu et al. ${ }^{[13]}$ who study CDX2 expression in different subtypes of intestinal metaplasia and gastric carcinoma and found CDX2 expression in $69.56 \%$ in IM foci associated with chronic atrophic gastritis (CAG), 53.13\% in IM foci and the expression of CDX2 protein was significantly lower in type III IM than in type I IM (46.42\% vs. $79.31 \%, \mathrm{P}<0.05)$.

Kang et al. ${ }^{[18]}$ also reported that CDX2 expression was significantly higher in incomplete type of IM than in complete type $(\mathrm{P}=0.045)$. But RongQin et al. ${ }^{[15]}$ showed no difference of CDX2 expression among type I, II and III IM.

As regarding CDX2 expression in gastric dysplasia, the present study showed $75 \%(6 / 8)$ expression in low-grade dysplasia and 50\% (2/4) in high dysplasia and positive CDX2 was significant in low -grade dysplasia These results are close to Liuet al. [13] who found CDX2 expression in $56 \%(9 / 16)$ for high grade dysplasia and $80 \%(12 / 15)$ for low grade dysplasia in their studied cases. But Kim et al. ${ }^{[19]}$ demonstrated a positive correlation between CDX2 expression and the increasing grade of dysplasia where CDX2 expression was detected in $73.3 \%$ of low-grade cases, $85.5 \%$ of high-grade.This discrepancy was due to smaller number in this study (12 cases) thanin their study (105 cases).

The results are different from RongQin et al. ${ }^{[15]}$ who demonstrate the CDX2 expression in 50\% (36/72), the positive rates of CDX2 were $43.5 \%$, $55 \%$ and $61.5 \%$ in mild, moderate and severe dysplasia, showing an increasing trend, with no relationship between the grade of gastric epithelial dysplasia and CDX2 expression, this discrepancy due to different clone; where in this study rabbit monoclonal was used while, in their study, they used mouse monoclonal antibody.

The results are close to Xin et al ${ }^{[14]}$ who found CDX2 expression in 51.61\% (48/93) of dysplasia regardless to the grade of dysplasia.

Li-Jun Xiao et al. ${ }^{[16]}$ also found CDX2 in $45.5 \%(25 / 55)$ of dysplasia.

As regarding CDX2 expression in gastric cancer, the present study showed that CDX2 expression is about $62.5 \%(5 / 8)$ for tubular adenocarcinoma (intestinal type), $100 \%(2 / 2)$ for mucinous carcinoma and $0 \%(0 / 2)$ for diffuse type( signet ring carcinoma).The expression of
CDX2 was also higher in intestinal-type than in diffuse-type gastric cancer. The result of the current study are close to these studies by Park Do et al ${ }^{[11]}$ who reported that CDX2 staining was observed in $61 \%$ of gastric adenocarcinomas and significantly favored in the intestinal-type tumors over the diffuse variants (77\% versus $45 \%$ ) but CDX2 expression in the present study in diffuse gastric cancer was $0 \%$.

The results are close toLiu et al. ${ }^{[13]}$ who found CDX2 expression in $42.50 \%$ in gastric carcinomas. The results are also agreed with Bai et al. ${ }^{[20]}$ who detect $\mathrm{CDX} 2$ expression in $55 \%$ $(38 / 69)$.

The results are close to Yang and Kim. ${ }^{[21]}$ who study the expression of CDX2 and p53 in 129 cases of gastric adenocarcinoma and found that $\mathrm{Cdx} 2$ was expressed in $51.7 \%$ of the gastric carcinomas. The $\mathrm{Cdx} 2$ was expressed more frequently in intestinal type than in diffuse type $(\mathrm{P}=0.005)$ and was expressed more frequently in moderate and well differentiated tumor than in poorly differentiated tumors $(\mathrm{P}=0.001)$.

The results go with seno et al ${ }^{[12]}$ who found CDX2 expression in $0 \%(0 / 12)$ for diffuse gastric cancer and 64\% (18/28) of intestinal-type gastric cancer and showed that CDX2 has been shown to be a key molecule associated with intestinal type gastric cancer. Xin et al. ${ }^{[14]}$ also found CDX2 expression in $61.54 \%$ (32/52) for gastric intestinal-type adenocarcinoma.

The results close to those obtained by Liuet al. ${ }^{[13]}$ that showed CDX2 expression in $67 \%$ (30/45) intestinal cancer but they found CXD2 expression in $42 \%(8 / 19)$ for diffuse. The difference may be due to smaller number of cases in the current study ( 2 cases of signet ring carcinoma).

The results are different from ReyhanBayrak et al. ${ }^{[22]}$ whofound CDX2 staining was observed in gastric adenocarcinomas $22.5 \%$ (16/71), more commonly in the intestinal-type than in the diffuse-type (28.9 vs $11.5 \%$ ) the difference due to smaller number of cases in the present study.

These results are close to Li-Jun Xiao et al. ${ }^{[16]}$ who found CDX2 expression in $76.0 \%$ $(19 / 25)$.

The results are close to Almeida et al. ${ }^{[19]}$ who found expression of CDX2 in 54\% (24/46) of gastric carcinomas.

In the current study, CDX2 expression in mucinous carcinoma was $100 \%(2 / 2)$. This is close to the results by Liuet al. ${ }^{[13]}$ that showed that CDX2 expressed in 83\% (5/6) of cases. 
In the present study, the expression of CDX2 in well and moderately differentiated adenocarcinoma (66.6\%) was higher than that in the poorly differentiated $50 \%$. This is in agreement withLiu et al. ${ }^{[13]}$ and RongQin et al. ${ }^{[15]}$ but Kim et al. ${ }^{[19]}$ show a statistically significant positive correlation between CDX2 and increasing grade of carcinoma.

The present study showed that CDX2 expression was decreased in gastric cancers, when compared with IM and dysplasia. This collective experience may suggest a potential tumor suppressor role for $\mathrm{CDX} 2$, in view of its sequential decrease in expression along with the stepwise gastric carcinogenesis (IM, epithelial dysplasia and gastric cancer). This opinion is shared by RongQin et al. ${ }^{[15]}$, Xin et al. ${ }^{[14]}$ and Liu et al. ${ }^{[13]}$ who showed that CDX2 expressions are progressively decreased in gastric IM, dysplasia and cancer.

The results of current study were in agree with Kang et al. ${ }^{[19]}$ who found that CDX1 and CDX2 may play an important role in the formation of IM and in the progression to dysplasia and GC in human gastric specimens using real-time polymerase chain reaction (RTPCR) method.

Song et al. ${ }^{[23]}$ reported a significantly better outcome for CDX2-positive gastric tumors over CDX2-negative tumors. Other studies have similarly demonstrated that positive CDX2 expression in gastric cancer significantly correlated with better differentiation and prognosis but this is not done in the present study.

No significant correlation between CDX2 and grade of the gastric cancer, this is different with Seno et al. ${ }^{[12]}$,Liu et al. ${ }^{[13]}$ and Bai et al $^{[17]}$ who found negative correlation between CDX2 and increasing grade of gastric cancer.

Intestinal metaplasia can be classified into different subtypes by several classification systems. The most widely accepted one is to classify IM into complete type and incomplete type, with the latter carrying a higher risk of gastric cancer.Reis et al. ${ }^{[24]}$.Type I intestinal metaplasia displays no expression of MUC6. De novo expression of MUC2 is conspicuous in goblet cells in type II and III intestinal metaplasia Reis et al. ${ }^{[24] ;}$ Babu et al ${ }^{[8]}$; Wang and Fang ${ }^{[25]}$.

High levels of MUC2 and mRNA and immune-reactive protein were found in specimens with intestinal metaplasiaHo et al. ${ }^{[26]}$. Different studies have supported the fact that alteration in the expression pattern of different mucins may contribute to alternation in epithelial cell growth, immune reaction, cellular adhesion and interaction with the extra-cellular matrix, which in turn may influence the tumorigenicity and metastatic properties of cancer cells Hollingsworth and Swanson $^{[27]}$.

Gastric carcinomas are classified into gastric and intestinal phenotype on the basis of mucin expression by surface mucous cells, glandular mucous cells and intestinal goblet cells. MUC2 is employed as a marker of intestinal goblet cell differentiation and MUC5AC and MUC6 as a marker of the gastric phenotype (diffuse type) (Machado et al. ${ }^{[30]}$; Pinto-de- Sousa et al. ${ }^{[7]}$.

As regarding MUC2 expression in chronic superficial gastritis and chronic atrophic gastritis, the present study showed that MUC2 negatively expressed in chronic superficial gastritis and chronic atrophic gastritis $0 \%(0 / 11)$ and this was in agree with Liu et al. ${ }^{[13]}$;Vernygorodskyi ${ }^{[31]}$;Babu et al. ${ }^{[8]}$ and RongQin et al. ${ }^{[15]}$.

In this study MUC2 was expressed in nearly all cases of intestinal metaplasia about $100 \%$ (15/15), 13 showing strong expression and 2 cases showing moderate expression. The expression of MUC2 was found in all goblet cells in all cases of complete intestinal metaplasia. MUC2 was expressed in mucous epithelial cells and in goblet cells in cases of incomplete intestinal metaplasia present in the cytoplasm and strongly in the cell membrane this is in agree with Reis et al. ${ }^{[24]}$ the same result obtained by Liu et al. ${ }^{[13]}(100 \%)$, Silberg et al. ${ }^{[9]}(100 \%)$ and with Babu et al. ${ }^{[8]}(>75 \%)$.

As regarding MUC2 expression in dysplasia, the present study showed MUC2 expression in about $75 \%$. MUC2 was expressed, in cytoplasm and strongly in the cell membrane of goblet cells or epithelial cells. The results close to results by Liuet al. ${ }^{[13]}$ who found MUC2 expression in $71 \%$ of cases.

In this study; significant difference was found as regarding MUC2 expression in low and high- grade dysplasia; where MUC2 was significant in low grade dysplasia $(\mathrm{p}<0.05)$ this is not in agree with Liu et al ${ }^{[13]}$ who found no significant difference in low and high grade dysplasia.

Regarding MUC2 expression in the current study in intestinal gastric cancer, MUC2 was expressed in about $50 \%$ this is close to the results of Liu et al. ${ }^{[13]} 51 \%$ (23/45) and to Geramizadeh et al. ${ }^{[32]}(38.2 \%)$ of their studied cases. The results close toKim et al. ${ }^{[33]}$ who found MUC2 expression in $35.4 \%$ (146 of 412). 
The same results obtained by Barresi et al. [8] who found MUC2 expression in 50\% of intestinal gastric cancer.

The results are different from that obtained byÖzgürillhan et al. ${ }^{[34]}$ who found MUC2 expression in $89.4 \%$ of intestinal gastric cancer.

MUC2 was expressed in 2/2 cases (100\%) of mucinous carcinoma and staticically significant. The results of this study reflected similar findings by Liuet al. ${ }^{[13]}$ who demonstrated that MUC2 expression in $6 / 6$ cases $(100 \%)$ this reflect intestinal differentiation. The same results obtained by Özgürİlhan et al. ${ }^{[34]}$ who found MUC2 expression in $100 \%$ of mucinous carcinoma.

In the present study, no positive immunostaining of MUC2 in patients with gastric signet-ring cell adenocarcinoma $0 \%(0 / 2)$ the same result obtained by Vernygorodskyi, ${ }^{[31]}$ but Liu et al. ${ }^{[13]}$ found MUC2 expression in diffuse cancer in $(58 \%)$ this discrepancy may be due to different sample size 2 cases in the present study and 11 in the other study.

As regarding MUC6 expression in the current study, it was expressed in all cases $100 \%$ (11/11) of chronic superficial gastritis and chronic atrophic gastritis; it was expressed in the cytoplasm of mucous neck cells of the body and deeper glands of the antrum. MUC6 expressed in the surface epithelium in patient with history of $\mathrm{H}$. pyloiinfection, the same result obtained by Vernygorodsky, ${ }^{[31]}$ and with Babu et al. ${ }^{[8]}$.

By mechanisms still unknown $\mathrm{H}$. pylori has been described to up- and downregulate MUC6 expression in the gastric epithelium Babu et al. ${ }^{[8]}$.

In this study, MUC6 was not expressed in complete intestinal metaplasia (0/3), $100 \%$ total negative; this is also confirmed by a study by Liu et al. ${ }^{[13]}$ and Vernygorodsky, ${ }^{[31]}$.

In the present study MUC6 was expressed in incomplete intestinal metaplasia in about $91.6 \%$ (11/12). The expression was strong in 9 cases and 2 cases showed moderate MUC6 expression. This is close to the result by Liuet al. ${ }^{[13]}$ and with Vernygorodsky, ${ }^{[31]}$ who found that MUC6 expression in all studied cases $100 \%$.

As regarding MUC6 expression, in the current study, in cases of dysplasia was about $16.6 \%(2 / 12)$ of all cases showing strong expression and statistically significant in low grade dysplasia. This opinion agreed by Liu et al. ${ }^{[13]}$ who found MUC6 expression in (19\%) and demonstrated that, the decreased expression of MUC6 indicates less differentiation and maturation of the cells in dysplasia.
Decreased expression of MUC6 in gastric carcinomas was confirmed in the current study. MUC6 expression in intestinal gastric cancer in the present study was $42 \%$ (5/8) and exhibits moderate to strong expression. The results were close to the result by Liu et al. ${ }^{[13]}$ who found MUC6 expression in (41\%) of their studied cases. The results were close to Khattab et al. ${ }^{[35]}$ who found MUC6 expression in 32\% (9/28) of cases.

The results were close to Saki et al. ${ }^{[36]}$ who found MUC6 expression in 48.4\% (46/95) of gastric cancer. Also close to that that obtained by Zang et al. ${ }^{[37]}$ who found MUC6 expression in (26.6\%) of their studied cases.

In this study, none of the mucinous types of gastric cancer were positive for MUC6 (0/2); $100 \%$ totally negative. This is agreeing with Daniela Lazăr1. ${ }^{[38]}$ and Liuet al. ${ }^{[13] .}$

The current study showed that MUC6 is expressed in $100 \%$ of diffuse (signet ring carcinoma) and was statistically significant, this is agreed by Liu et al. ${ }^{[13]}$ and Vernygorodsky, ${ }^{[31]}$. This indicates that MUC6 is amarker of diffuse type of gastric carcinoma.

In this study, the role of using CDX2 and MUC2 antibodies were evaluated together in chronic superficial gastritis and chronic atrophic gastritis characteristic and statistically significant IHC profiles was observed (CDX2-/MUC2-) this is in agree withLiuet al. ${ }^{[13]}$.

In intestinal metaplasia a characteristic and statistically significant IHC profiles was observed (CDX2+/MUC2+) and this indicate that these two markers are markers of intestinal metaplasia. This is in agree with KristinaRoessler et al. ${ }^{[39]}$ who demonstrated that CDX2 and MUC2 expression correlated positively and $\mathrm{CDX} 2$ acts as a transcription factor of intestinal gene products like MUC2 by binding of CDX2 to a special sequence of the MUC2 promoter this is confirmed by astudy by Yamamoto et al. ${ }^{[40]}$.

\section{REFERENCES}

1-Jemal A., Bray F., Center M.M., Ferlay J., Ward E., Forman D. (2011): Global cancer statistics. CA Cancer J Clin; 61: 69-90.

2-El- Bolkainy M.N., Nouh M.A., Farahat I.G., ElBolkainy T.N., Badawy O.M. (2013). Gastro intestinal cancer in pathology of cancer, the National Cancer Institute, Cairo University.4th edition chapter 13, pp. 147-230.

3-Correa P and Piazuelo M.B. (2012): The gastric precancerous cascade. J Dig Dis; 13: 2-9.

4-Peek R. M. and Crabtree J. E. (2006): Helicobacter infection and gastric neoplasia. J Pathol; 208: 233- 248.

5-González C.A., Pardo M.L., Liso J.M., et al. (2010): Gastric cancer occurrence in preneoplastic 
lesions: a long-term follow-up in a high-risk area in Spain. Int J Cancer; 127:2654-2660.

6-Correa P and Hougton I. (2007): The gastric dysplasia. J Dig Dis; 12: 3-9.

7-Pinto-de-Sousa J., David L., Reis C. A., Gomes R., Silva L., and Pimenta A. (2002): Mucins MUC1, MUC2, MUC5AC and MUC6 expression in the evaluation of differentiation and clinicobiological behaviour of gastric carcinoma. Virchows Arch; 440: 304- 310.

8-Babu S. D., Jayanthi V., Devaraj N., Reis C. A., and Devaraj H. (2006): Expression profile of mucins (MUC2, MUC5AC and MUC6) in Helicobacter pylori infected pre-neoplastic and neoplastic human gastric epithelium. Mol Cancer; 5: 10-13.

9-Silberg D.G., Swain G.P., Suh E.R., andTraber P.G. (2000): CDX1 and CDX2 expression during intestinal development. Gastroenterol; 119: 961971.

10-Fan Z., Li J., Dong B., Huang X. (2005): Expression of CDX2 and hepatocyte antigen in gastric carcinoma: correlation with histologic type and implications for prognosis. Clin Cancer Res; 11: 6162-6170.

11-Park Do Y., Srivastava A., and Kim G.H., et al. (2010): CDX2 expression in the intestinal-type gastric epithelial neoplasia: frequency and significance. Mod Pathol; 23: 54-61.

12-Seno H., Oshima M., Taniguchi M.A., et al. (2002): CDX2 expression in the stomach with intestinal metaplasia and intestinal-type cancer: Prognostic implications. Int J Oncol; 21: 769-774.

13-Liu Q., Teh M., Ito K., Shah N., Ito Y., Yeoh K., G. (2007): CDX2 expression is progressively decreased in human gastric intestinal metaplasia, dysplasia and cancer. Mod Pathol; 20: 1286-1297.

14-Xin S., Huixin C., Benchang S., et al. (2007): Expression of CDX2 and claudin-2 in the multistage tissue of gastric carcinogenesis. Oncology; 73: 357-365.

15-Rong Qin, Na-Na Wang, Jing Chu and Xian Wang. (2012): Expression and significance of homeodomain protein CDX2 in gastric carcinoma and precancerous lesions. World J Gastroenterol;18(25): 3296-3302.

16-Li-Jun Xiao, Shuang Zhao, En-Hong Zhao., et al.(2012):Clinicopathological and Prognostic Significance of MUC- 2, MUC-4 and MUC-5AC Expression in Japanese Gastric Carcinomas.Asian Pacific Journal of Cancer Prevention;13: 64476451.

17-Almeida R., Silva E., Santos-Silva F., et al. (2003): Expression of intestine-specific transcription factors, CDX1 and CDX2, in intestinal metaplasia and gastric carcinomas. J Pathol; 199: 36- 40.

18-Kang J.M., Lee B.H., Kim N., et al. (2011):CDX1 and CDX2 expression in intestinal metaplasia, dysplasia and gastric cancer. J Korean Med Sci; 26:647-653.

19-Kim H.S., Lee J.S., Freund J.N., Min K.W., Lee J.S., Kim W., Juhng S.W., Park C.S. (2006): CDX-2 homeobox gene expression in human gastric carcinoma and precursor lesions. J GastroenterolHepatol; 21: 438-442.

20-Bai Y.Q., Yamamoto H., Akiyama Y.,et al. (2002): Ectopic expression of homeodomain protein CDX2 in intestinal metaplasia and carcinomas of the stomach. Cancer Lett; (9):13.

21-Yang and Kim (2011): expression of CDX2 and p53 in gastric carcinoma. GUT;5:532.

22-Reyhan Bayrak, HacerHaltas and SibelYenidunya. (2012): The value of CDX2 and cytokeratins 7 and 20 expression in differentiating colorectal adenocarcinomas from extraintestinal gastrointestinal adenocarcinomas: cytokeratin 7 $120+$ phenotype is more specific than CDX2 antibody. Diagnostic Patholo;7:9.

23-Song J.H., Kim C.J., Cho Y.G., et al. (2008): Genetic alterations of the CDX2 gene in gastric cancer.APMIS; 116: 74-80.

24-Reis C. A., David L., Correa P., et al. (1999): Intestinal metaplasia of human stomach displays distinct patterns of mucin (MUC1, MUC2, MUC5AC, and MUC6) expression. Cancer Res; 59: 1003- 1007.

25-Wang R.Q., and Fang D., C. (2003). Alterations of MUC1 and MUC3 expression in gastric carcinoma: relevance to patient clinicopathological features. J ClinPathol, 56, 378-84.

26-Ho S. B., Shekels L. L., Toribara N. W., et al. (1995): Mucin gene expression in normal, preneoplastic, and neoplastic human gastric epithelium. Cancer Res; 55: 2681- 2690.

27-Hollingsworth M. A. and Swanson B. J. (2004): Mucins in cancer: protection and control of the cell surface. Nat Rev Cancer; 4: 45- 60.

30-Machado J.C., Nogueira A. M., Carneiro F., Reis C. A., and Sobrinho- Simoes M. (2000): Gastric carcinoma exhibit distinct types of cell differentiation: an immunohistochemical study of trefoil peptides (TFF1 and TFF2) and mucins (MUC1, MUC2, MUC5AC, and MUC6). J Pathol; 190: 437- 443.

31-Vernygorodskyi S., $\quad$ V. (2013): Immunohistochemical Profile of Mucins and their Expression in Precancerous Changes of the Stomach.International Journal of BioMedicine $3(2) ; 115-118$.

32-Geramizadeh B., Mokhtari M., Sefidbakht S., and Rahsaz M. (2012): Mucin Profile Expression in Gastric Adenocarcinoma. Middle East J Dig Dis; 4:211-5

33-Kim Y., Nari Shin, Gwang Ha Kim.et al. (2013): Mucin Expression in Gastric Cancer. Reappraisal of Its Clinicopathologic and Prognostic Significance. Arch Pathol Lab Med; 137:10471053.

34-Özgür İLHAN, Ünsal HAN, Binnur ÖNAL, SerkanYaşar ÇELIK. (2012): Prognostic significance of MUC1, MUC2 and MUC5AC expressions in gastric carcinoma; 21 (4): 345-352.

35-Kattab M., A., Wesam A., and Lofty M. (2010): MUC2 and MUC6 apomucins expression in 
human gastric neoplasm: an immunehistochemical analysis. Med Oncol 28: S207-S213.

36-Saki H., Eshi y., li X.1., and Akayama (2008): Pdx1 homobox protein expression in pseudopyloric glands and gastric carcinoma.Gut;53(3): 1-8.

37-Zhang Xia, Tetsuya Tsukamoto, TstomuMizoshitaet et al., (2009): expression of osteopontin and CDX2: Indications of phenotypes and prognosis in advanced gastric cancer Oncol Rep 21: 609613.
38-DanielaLazăr. (2013): "Gastric Carcinoma- New Insights into Current management". Gastroenterol. ISBN; 978-953-51-0914-3.

39-Kristina Roessler, Stefan P., Mqing Paul M. et al. (2005): Co-expression of CDX2 and MUC2 in gastric carcinomas: correlations with clinicopathological parameters and prognosis.World $\mathbf{J}$ Gastroenterol; 11(21):3182-3188.

40-Yamamoto H., Bai Y.Q. and Yuasa Y. (2003): Homeodomain protein $\mathrm{CDX} 2$ regulates gobletspecific MUC2 gene expression. BiochemBiophys Res Commun; 300: 813-818. 